\title{
3D-TEM study of gamma alumina catalyst supports
}

\author{
Wharton Sinkler*, Steven A. Bradley * Ulrike Ziese ** and Krijn P. de Jong *** \\ * UOP LLC, 25 E. Algonquin Rd., Des Plaines IL 60017-5017 \\ **Kavli Institute of NanoScience, National Center HREM, Delft University of Technology \\ Lorentzweg 1, 2628 CJ Delft, The Netherlands \\ *** Department of Inorganic Chemistry and Catalysis, Debye Institute, Utrecht Uninversity, \\ Sorbonnelaan 16, 3584 CA Utrecht, The Netherlands
}

Gamma alumina is one of the most widely used catalyst supports for commercial catalytic applications. In addition to factors such as low cost and mechanical strength, the features which are responsible for the wide application of gamma alumina are its high surface area and large volume of open mesoporosity, which allows feed molecules to penetrate rapidly and uniformly to catalytic sites. While porosimetry can provide quantitative measurement of pores size distributions, subtle features of the pore geometry are determined by changes to the morphologies of gamma alumina crystallites with treatments such as calcinations and steaming. The grain geometries may also influence chemistry due to shifts in relative quantities of different types of site which are available for anchoring catalytic metal particles and adsorbing/desorbing feed molecules and products. Because grain sizes of gamma alumina used in commercial applications are typically in the range of 5-50 nm, deducing the 3-dimensional geometry by standard TEM or SEM is not straightforward. 3D-TEM provides an ideal means for a more complete and potentially quantifiable approach to assessing pore and particle geometries, and how these vary by thermal treatments. While previous publications have applied 3D-TEM to alumina supports [1], this presentation will demonstrate the feasibility of detecting differences in grain morphologies arising from different heat treatments.

The starting material was a sol-gel alumina with BET surface area of $218 \mathrm{~m}^{2} / \mathrm{g}$. Two thermally treated aluminas were also used, one involving steam the other a dry calcination; conditions were adjusted such that the final BET was $180 \mathrm{~m}^{2} / \mathrm{g}$ in both cases. For 3D-TEM imaging, microtomed thin sections were made after embedding coarse powders of the materials in sulfur. Sections were collected on holey carbon films to which $5 \mathrm{~nm}$ gold fiducial markers had been previously applied. The sulfur was sublimed off in vacuum prior to data collection. Tilt series of 141 images at $1^{\circ}$ intervals ( \pm 70 degrees of tilt) were acquired on a $200 \mathrm{kV}$ Tecnai FEG microscope equipped with a CCD camera. Alignment and generation of tomograms was performed with IMOD software [2].

Figures 1-3 show sections through tomograms for each of the samples. The sections represent slices through approximately the middle of the thin section samples. For comparison, Fig. 4 shows a single image taken from the tilt series used to generate Fig. 2. Comparing Figs. 2 and 4 it can be seen that the tomographic section provides far superior interpretability of pore and particle geometry than is the case for the TEM image, because in the latter case many particles are superimposed. The effects of steaming and calcination are evident in thickening of the alumina plates and a reduction of aspect ratio with respect to the starting material. The aspect ratio appears often smaller in the dry calcined case than in the steamed (Figs. 2 and 3), but the steamed case also has significantly smoother and more regular grains. In contrast, the particles in the dry calcined alumina have rougher surfaces. The increased roughness may compensate for the reduced aspect ratio, so BET 
surface areas are the same for both steamed and dry calcined samples in spite of quite different overall geometries.

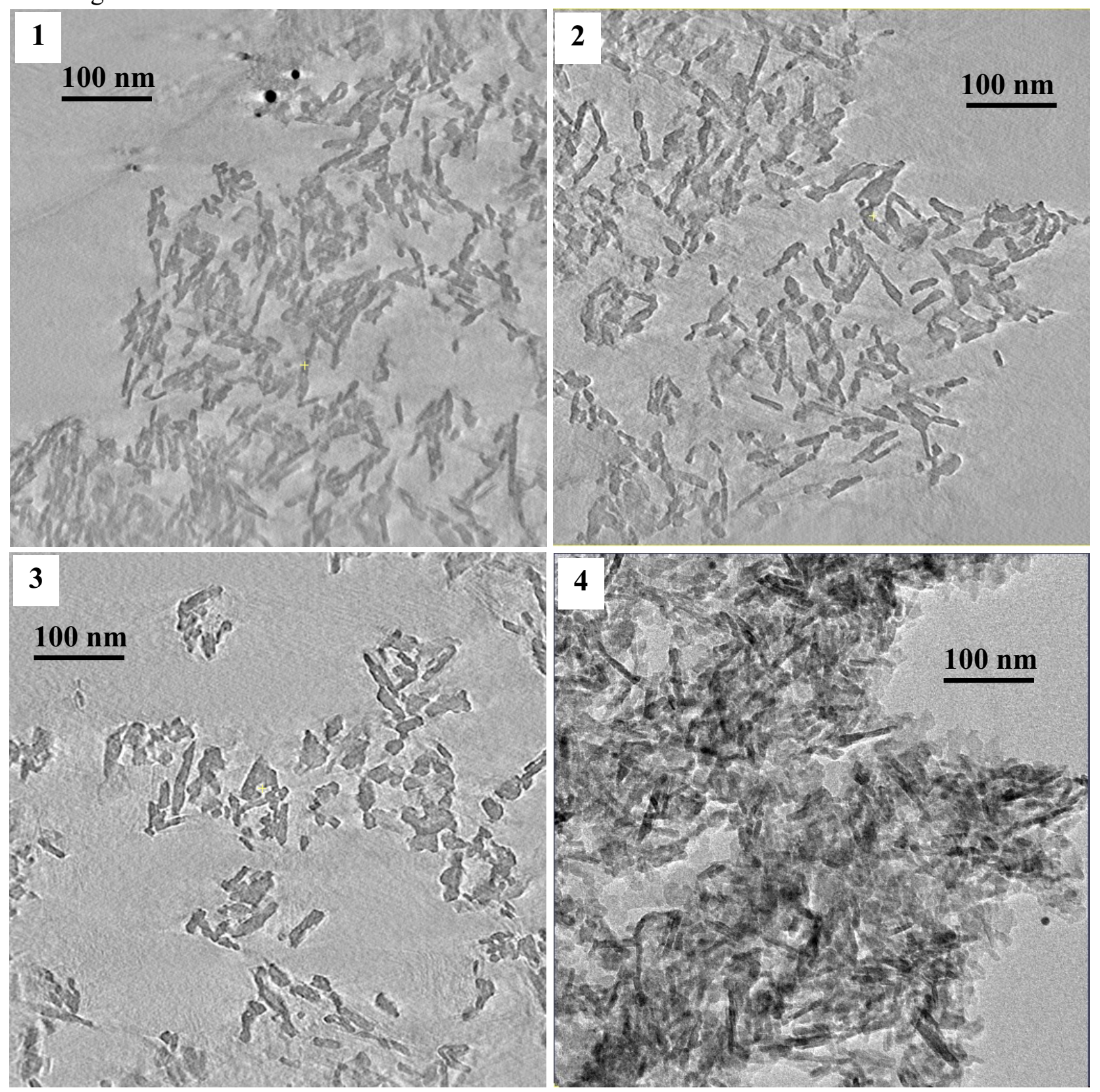

Fig. 1. Tomographic section through sample of the starting alumina. Fig. 2. Tomographic section through steamed material. Fig. 3. Tomographic section through dry calcined alumina. Fig. 4. Standard TEM image taken from the tilt series used to generate Fig. 2.

\section{References}

[1] C. Kübel et al., Microsc. Microanal. 11 (2005) 378.

[2] Kremer J.R., D.N. Mastronarde and J.R. McIntosh J. Struct. Biol 116 (1996) 71. http://bio3d.colorado.edu/imod/. 